R. V. Gurjar, C. R. Pradeep and D.-Q. Zhang

Nagoya Math. J.

Vol. 168 (2002), 41-63

\title{
ON GORENSTEIN SURFACES DOMINATED BY $\mathbf{P}^{2}$
}

\author{
R. V. GURJAR, C. R. PRADEEP AND D.-Q. ZHANG
}

\begin{abstract}
In this paper we prove that a normal Gorenstein surface dominated by $\mathbf{P}^{2}$ is isomorphic to a quotient $\mathbf{P}^{2} / G$, where $G$ is a finite group of automorphisms of $\mathbf{P}^{2}$ (except possibly for one surface $V_{8}^{\prime}$ ). We can completely classify all such quotients. Some natural conjectures when the surface is not Gorenstein are also stated.
\end{abstract}

\section{$\S 1$. Introduction}

Let $V$ be a normal projective surface defined over C. $V$ is said to be a log del Pezzo surface if $V$ has at worst quotient singularities and the anti-canonical divisor $-K_{V}$ is ample. The rank of $V$ is the Picard number $\rho(V)=\operatorname{dim}_{\mathbf{Q}} \operatorname{Pic}(V) \otimes \mathbf{Q}$. It is easy to see that any quotient of $\mathbf{P}^{2}$ by a finite group of automorphisms is a log del Pezzo surface of rank one. Miyanishi and Zhang have raised the question of giving a criterion for a projective normal surface to be isomorphic to $\mathbf{P}^{2} / G$, where $G$ is a finite group of automorphisms of $\mathbf{P}^{2}$. In [9] certain rank 1 log del Pezzo surfaces are shown to be quotients of $\mathbf{P}^{2}$ modulo a finite group.

Our main theorem is the following result.

Theorem 1. Let $V$ be a Gorenstein normal surface and let $f: \mathbf{P}^{2} \rightarrow$ $V$ be a non-constant morphism. Then we have the following assertions:

(1) If $\pi_{1}(V \backslash \operatorname{Sing} V)$ is trivial then $V$ is isomorphic to one of the following surfaces.

(i) The projective plane $\mathbf{P}^{2}$,

(ii) The quadric cone $Q:=\left\{X^{2}+Y^{2}+Z^{2}=0\right\}$ in $\mathbf{P}^{3}$,

(iii) A surface of singularity type $A_{1}+A_{2}$, or

(iv) The surface $V_{8}^{\prime}$ which has a unique singular point, which is analytically the $E_{8}$ singular point. (cf. Theorem 2 below).

Received June 28, 2001.

2000 Mathematics Subject Classification: 14J25, 14J26, 14J45. 
(2) If $\pi_{1}(V \backslash \operatorname{Sing} V)$ is non-trivial then $f$ factors as $\mathbf{P}^{2} \rightarrow W \rightarrow V$, where $W$ is one of the surfaces (i) or (ii) in (1) above and $W \rightarrow V$ is étale over $V \backslash \operatorname{Sing} V$.

(3) $V$ is always isomorphic to a quotient $\mathbf{P}^{2} / G$ for a finite group of automorphisms of $\mathbf{P}^{2}$, except for the surface $V_{8}^{\prime}$ in the case (iv) above. The surface $V_{8}^{\prime}$ is not isomorphic to any quotient of $\mathbf{P}^{2}$ modulo a finite group of automorphisms.

Remarks. (1) We can give a very precise description of any $V$ in part (3) above, particularly its singularity type and $\pi_{1}(V \backslash \operatorname{Sing} V)$ and the corresponding surface $W$ as in part (2) above. (See Section 5.)

(2) The surfaces $V_{8}, V_{8}^{\prime}$ are "twin" surfaces. Theorem 1 says that there is no non-constant morphism $\mathbf{P}^{2} \rightarrow V_{8}$. It is most probable that there is no such map $\mathbf{P}^{2} \rightarrow V_{8}^{\prime}$ but we have been unable to prove this. This is the exception in part (iv) above.

We will also prove the following result which will be used in the proof of Theorem 1. This result is stated in [9] and a sketch of proof is given there. In view of the importance of this result for our proof of Theorem 1 we will give a complete proof. We would like to point out that the uniqueness assertion made in Lemma 7 of [9] is not quite correct. For $K_{V}^{2}=1$ we have found two non-isomorphic surfaces.

Theorem 2. Let $V$ be a Gorenstein log del Pezzo surface of rank 1 such that $\pi_{1}(V \backslash \operatorname{Sing} V)=(1)$. Let $d:=\left(K_{V}\right)^{2}$. Then we have the following assertions:

(i) $1 \leq d \leq 9$ and $d=9$ implies $V \cong \mathbf{P}^{2}$. If $d=1$ then $V$ is one of the following two surfaces in the weighted projective space $\mathbf{P}(1,1,2,3)$.

$$
\begin{gathered}
V_{8}:\left\{W^{2}+Z^{3}+X^{5} Y=0\right\} . \\
V_{8}^{\prime}:\left\{W^{2}+Z^{3}+X^{5} Y+X^{4} Z=0\right\} .
\end{gathered}
$$

Both these have a unique singularity of type $E_{8}$. The surface $V_{8}$ contains a rational curve $C$ with only one ordinary cusp (and otherwise smooth) such that $C \sim-K_{V}$. The surface $V_{8}^{\prime}$ contains a rational curve $C$ with only one ordinary double point (and otherwise smooth) such that $C \sim-K_{V}$. In both these cases $C$ does not pass through the singular point of $V$. 
(ii) If $d>1$, then $V$ contains a cuspidal rational curve $C$ as in (i) above such that $C \sim-K_{V}$ and $C$ does not pass through the singular point of $V$. The surface $V$ is uniquely determined by the integer $d$.

(iii) If $d \neq 8$ then $V$ contains an irreducible curve $\Delta$ such that $\Delta$ generates the Weil divisor class group of $V$ and $-K_{V} \sim d \Delta$. If $d=8$ then $V$ contains an irreducible curve $\Delta$ which generates the Weil divisor class group of $V$ and $-K_{V} \sim 4 \Delta$.

(iv) In case $1<d \leq 5$ or $d=1$ and $V=V_{8}$ the affine surface $V \backslash C$ is isomorphic to $\mathbf{C}^{2} / G$, where $G$ is a finite group of automorphisms of $\mathbf{C}^{2}$ isomorphic to the fundamental group at infinity of $V \backslash C$.

We now mention several results proved by other mathematicians which are closely related to our Theorems 1 and 2 .

(1) Demazure has proved important results about Gorenstein log del Pezzo surfaces in [3], particularly about the linear systems $\left|-n K_{V}\right|$.

(2) In [4] general results about embeddings of Gorenstein log del Pezzo surfaces are proved.

(3) In [9] a classification of rank 1 Gorenstein log del Pezzo surfaces is given.

(4) In [7] R. Lazarsfeld has proved that any smooth variety which is dominated by $\mathbf{P}^{n}$ is isomorphic to $\mathbf{P}^{n}$.

(5) In [5] and [8] it is proved that if there is a proper map $f: \mathbf{C}^{2} \rightarrow V$ onto a normal algebraic surface $V$ then $V$ is isomorphic to $\mathbf{C}^{2} / G$ for a finite group of automorphisms of $\mathbf{C}^{2}$.

(6) In [6], Mohan Kumar has shown that if a normal rational surface $S$ has a singularity of type $E_{8}$ then the local ring of $S$ at this singular point is isomorphic to one of the (non-isomorphic) local rings, viz. $\mathbf{C}[X, Z, W]_{(X, Z, W)} /\left(W^{2}+Z^{3}+X^{5}\right)$ or, $\mathbf{C}[X, Z, W]_{(X, Z, W)} /\left(W^{2}+Z^{3}+\right.$ $\left.X^{4} Z+X^{5}\right)$. This result will be used in Section 3 .

(7) In [1] it is proved that a surface of the form $\mathbf{P}^{2} / G$ cannot have a unique singular point of the type $E_{6}, E_{7}$ or $E_{8}$. This is a special case of our Theorem 1.

Our proof of Theorem 1 is almost self-contained. We do not use the classification mentioned in (3) above. All we need is some general results about embeddings given by $\left|-K_{V}\right|$ and $\left|-2 K_{V}\right|$ which are proved in [4]. Nevertheless, the paper [9] has been important for us while thinking of the proofs in this paper.

Recently, the first named author has proved the following general result using the results of this paper in an important way. 
Let $\pi: \mathbf{P}^{2} \rightarrow \mathbf{P}^{2}$ be a non-constant morphism. Let $C$ be an irreducible curve of degree $>1$ in $\mathbf{P}^{2}$ which is ramified for $\pi$. Then the greatest common divisor of the ramification indices of the irreducible curves lying over $C$ is 1 . In particular, $\pi^{-1}(C)$ cannot be irreducible.

\section{§2. Preliminaries}

All the algebraic varieties we consider are defined over the field $\mathbf{C}$ of complex numbers. A smooth complete rational curve $C$ on a smooth algebraic surface $S$ is called a $(-n)$-curve if $C^{2}=-n$. Let $Z$ be an irreducible normal variety such that $\pi_{1}(Z \backslash \operatorname{Sing} Z)$ is finite. Let $W^{\prime}$ be the universal covering space of $Z \backslash \operatorname{Sing} Z$. Then $W^{\prime}$ is also a variety. The normalization, $W$, of $Z$ in the function field of $W^{\prime}$ is called the quasi-universal cover of $Z$. There is a proper morphism with finite fibers $W \rightarrow Z$ which is unramified over $Z \backslash \operatorname{Sing} Z$. For any normal variety $Z$ we will denote the Zariski-open subset $Z \backslash \operatorname{Sing} Z$ by $Z^{0}$. Let $Z$ be an irreducible normal variety. An algebraic action of $\mathbf{C}^{*}$ on $Z$ is said to be a $\operatorname{good} \mathbf{C}^{*}$-action if $Z$ contains a point $z$ which is in the closure of every orbit. In this case we also say that $Z$ is a quasi-homogeneous variety.

In this section we collect a few results which we will use (at least implicitly) to prove Theorems 1 and 2. The following is from lemma 6 of [9]. Recall that $d:=K_{V}^{2}$. The integer $d$ is called the degree of $V$.

Lemma 1. (Reproved in Theorem 2) Let $V$ be a Gorenstein log del Pezzo surface of rank one and let $V^{\circ}$ denote the smooth locus of $V$. If $\pi_{1}\left(V^{\circ}\right)$ is trivial then $V$ has one of the following combinations of singularities: $A_{1}, A_{1}+A_{2}, A_{4}, D_{5}, E_{6}, E_{7}, E_{8}$. The values of $K_{V}^{2}$ in these cases are $8,6,5,4,3,2,1$ respectively. (Note that the case $K_{V}^{2}=7$ does not occur.)

Here $A_{1}+A_{2}$ means that there are two singular points on $V$, one of type $A_{1}$ and the other of type $A_{2}$. We will call these as the Dynkin types of $V$. Sometimes we also say that $V$ is of type $A_{1}, A_{2}$.

The following result plays an important role in our proof (see Corollary 4.5 of [4]):

Lemma 2. Let $V$ be a Gorenstein log del Pezzo surface of degree d. If $d \geq 3$ then the linear system $\left|-K_{V}\right|$ is very ample and gives a projectively normal embeding of $V$ in $\mathbf{P}^{d}$. 
If $d=2$ then $\left|-2 K_{V}\right|$ is very ample and gives a projectively normal embedding of $V$ in $\mathbf{P}^{6}$.

We will need the following result in our proof (see Lemma 6.1 of [11]).

Lemma 3. Let $G$ be an algebraic group which acts algebraically on a normal variety $Y$. Suppose $f: Z \rightarrow Y$ is a finite morphism with $Z$ a normal variety. If $Z$ contains a non-empty $G$-equivariant Zariski-open subset $Z_{0}$ such that $f$ restricted to $Z_{0}$ is $G$-equivariant then there is a unique action of $G$ on $Z$ such that $f$ is a $G$-morphism.

\section{§3. Proof of Theorem 2}

In this section we will prove Theorem 2 (cf. Introduction). This and some of the arguments in the proof of Theorem 2 will be used in proving Theorem 1. So let $V$ be a Gorenstein log del Pezzo surface of rank 1 such that $\pi_{1}(V \backslash \operatorname{Sing} V)$ is trivial.

Let $g: \tilde{V} \rightarrow V$ be a minimal resolution of singularities. Then $K_{\tilde{V}}^{2}=$ $K_{V}^{2}>0$. By assumption, $\left|n K_{\tilde{V}}\right|=\phi$ for $n>0$. Since $\pi_{1}(V \backslash \operatorname{Sing} V)$ is trivial the surface $\tilde{V}$ is simply-connected. Now it follows by Noether's theorem that $\tilde{V}$ is rational. Hence $K_{\tilde{V}}^{2}+b_{2}(\tilde{V})=10$. This implies that $1 \leq K_{\tilde{V}}^{2} \leq 9$. If $b_{2}(\tilde{V})=1$ then $V$ is smooth and hence isomorphic to $\mathbf{P}^{2}$. From now onwards, we will assume that $1 \leq K_{\tilde{V}}^{2} \leq 8$. We will assume in what follows that $V$ is not smooth. Consider first the case $d=8$. Then $\tilde{V}$ contains a $(-2)$-curve $C$. This implies that $\tilde{V}$ is the Hirzebruch surface $\Sigma_{2}$ and $V$ is obtained by contracting the $(-2)$-curve to an $A_{1}$-singularity. In this case $V$ is isomorphic to the quadric cone $Q:=\left\{X^{2}+Y^{2}+Z^{2}=0\right\}$ in $\mathbf{P}^{3}$. Let $x, y, z$ denote suitable homogeneous coordinates on $\mathbf{P}^{2}$. Then the group $G:=\mathbf{Z} /(2)$ acts on $\mathbf{P}^{2}$ by sending $[x, y, z] \rightarrow[-x,-y, z]$. The line at infinity $\{z=0\}$ is pointwise fixed and we see easily that $\mathbf{P}^{2} /(G)$ is isomorphic to $Q$. Then Theorem 2 (ii), (iii) are clear.

Claim. The case $d=7$ cannot occur.

To see this, assume that $d=7$. Then $\tilde{V}$ is obtained from a Hirzebruch surface $\Sigma_{n}$ by one blowing-up with $E$ the exceptional curve. Let $S$ be the unique curve with self-intersection $-n \leq 0$ on $\Sigma_{n}$ and $L$ be a fiber of the $\mathbf{P}^{1}$-fibration on $\Sigma_{n}$. We can assume that the blown-up point $p \in L$. We have the formula $K_{\Sigma_{n}} \sim-2 S-(2+n) L$. If $p \notin S$ then $K_{\tilde{V}} \sim-2 S-(2+n) L^{\prime}-$ 
$(1+n) E$, where $L^{\prime}$ is the proper transform of $L$. Clearly, $\tilde{V}$ contains a $(-2)$ curve different from $S$, say $C$. Since $K \cdot C=0$, the curve $C$ is disjoint from $S, L, E$. This is impossible. If $p \in S$ then $K \sim-2 S-(2+n) L^{\prime}-(2+n) E$. Again there is a $(-2)$-curve $C$ different from $S$. We get a contradiction as above.

In view of these observations, for the rest of the section we assume that $1 \leq d \leq 6$. First we will give a construction of such surfaces with $1 \leq d \leq 6$ and later on prove that these are all the surfaces we are looking for. We will construct examples of rank 1, Gorenstein log del Pezzo surfaces $V_{i}, 3 \leq i \leq 8$, and a rank 1, Gorenstein log del Pezzo surface $V_{8}^{\prime}$ such that $K_{V_{i}}^{2}=9-i, K_{V_{8}^{\prime}}^{2}=1$, and of singularity types $A_{1}+A_{2}, A_{4}, D_{5}, E_{6}, E_{7}, E_{8}, E_{8}$ respectively. Moreover, $V_{i}, V_{8}^{\prime}$ are compactification of $\mathbf{C}^{2}$ with an irreducible boundary curve. In particular, these surfaces have simply-connected smooth parts.

Let $X$ (resp. $X^{\prime}$ ) be a relatively minimal rational elliptic surface with a unique section $E$ (see Claim 2 in Lemma 4) and singular fibres of types $I I^{*}, I I$ (resp. $\left.I I^{*}, I_{1}, I_{1}\right)$. Such $X$ (resp. $X^{\prime}$ ) is unique modulo fibrationpreserving isomorphisms. The construction and uniqueness is shown by letting $X \rightarrow \mathbf{P}^{2}$ (resp. $X^{\prime} \rightarrow \mathbf{P}^{2}$ ) be the composition of blow-downs of the section $E$ and all components in the type $I I^{*}$ fibre except for a multiplicity 3 component $C_{3}^{\prime}$. We get a pencil in $\mathbf{P}^{2}$ generated by a cuspidal cubic and three times the tangent line at an inflexion point (resp. a pencil generated by a nodal cubic and three times the tangent line at an inflexion point). The pair of a cuspidal (resp. nodal) cubic curve and the tangent line at an inflexion point is unique upto projective transformations. Write the type $I I^{*}$ fibre as $\sum_{i=1}^{6} i C_{i}+4 C_{4}^{\prime}+2 C_{2}^{\prime}+3 C_{3}^{\prime}$ so that $\sum_{i=1}^{6} C_{i}+C_{4}^{\prime}+C_{2}^{\prime}$ is an ordered linear chain.

Let $X \rightarrow V_{8}$ (resp. $X^{\prime} \rightarrow V_{8}^{\prime}$ ) be the contraction of $E$ and the type $E_{8}$ divisor in the type $I I^{*}$ fibre to a smooth point and a singularity of type $E_{8}$. Then $V_{8}$ and $V_{8}^{\prime}$ are two non-isomorphic rank 1, Gorenstein log del Pezzo surfaces of singularity type $E_{8}$. Let $\Delta$ be the image on $V_{8}$ or $V_{8}^{\prime}$ of $C_{1}$.

For $3 \leq i \leq 8$ (resp. $i=1$ ), we let $X \rightarrow V_{i}$ be the contraction of $E$ and all components in the type $I I^{*}$ fibre, except $C_{9-i}$ (resp. $\left.C_{4}^{\prime}\right)$; denote by $\Delta$ the image on $V_{i}$ of $C_{9-i}$ (resp. $\left.C_{4}\right)$.

We will show that $V_{i}, V_{8}^{\prime}$ are as described above. Denote by $C$ the image on $V_{i}$ (resp. $V_{8}^{\prime}$ ) of the fibre of type $I I$ (resp. $I_{1}$ ). Then one has

$$
-K_{V} \sim C \sim(9-i) \Delta, V=V_{i}, V_{8}^{\prime}(i \neq 1) \text { and }-K_{V} \sim 4 \Delta \text { for } i=1 \text {, }
$$


where $\Delta$ is the generator of the Weil divisor class group $\operatorname{Div}(V)$. The last assertion here comes from the observation that the lattice on $X$ or $X^{\prime}$ which is generated by the section $E$, an elliptic fibre and the type $E_{8}$ sublattice in the type $I I^{*}$ fibre, is unimodular and hence equals $\operatorname{Div}(X)$ or $\operatorname{Div}\left(X^{\prime}\right)$.

By Kodaira's canonical bundle formula we have $K_{X} \sim-F$, where $F$ is any scheme-theoretic fiber of the elliptic fibration. This implies that $K_{V_{i}} \sim-C$ and hence $-K_{V_{i}}$ is ample. Similarly $-K_{V_{8}^{\prime}}$ is ample.

When $i=3$, the type $A_{1}, A_{2}$ singular points of $V_{3}$ lie on the smooth rational curve $\Delta$; when $4 \leq i \leq 8$, each of $V_{i}$ and $V_{8}^{\prime}$ has the unique singularity at the cusp of the cuspidal rational curve $\Delta$.

We assert that $V_{i} \backslash \Delta$ and $V_{8}^{\prime} \backslash \Delta$ are all isomorphic to the affine plane $\mathbf{C}^{2}$. Indeed, $V_{i} \backslash \Delta=V_{8} \backslash \Delta$, and hence we have only to consider $V_{8} \backslash \Delta$ $\left(V_{8}^{\prime} \backslash \Delta\right.$ is similar $)$. Now $S_{0}=2\left(E+\sum_{i=1}^{6} C_{i}\right)+C_{3}^{\prime}+C_{4}^{\prime}$ is the unique singular fibre of a $\mathbf{P}^{1}$-fibration $\varphi: X \rightarrow \mathbf{P}^{1}$ with $C_{2}^{\prime}$ as a section. The assertion follows from the observation that $V_{8} \backslash \Delta=X \backslash\left(S_{0}+C_{2}^{\prime}\right) \cong \mathbf{C}^{2}$.

LEMMA 4. (1) $V_{8}$ and $V_{8}^{\prime}$ are not isomorphic to each other.

(2) Every rank 1, Gorenstein log del Pezzo surface $V$ satisfying $1 \leq$ $K_{V}^{2} \leq 6$ with $\pi_{1}\left(V^{0}\right)=(1)$ is isomorphic to one of the 7 surfaces $V_{i}(3 \leq$ $i \leq 8), V_{8}^{\prime}$.

Proof. Some of the results and arguments in the proof below are wellknown to the experts, but we are giving them for the sake of completeness. At any rate, the assertion in part (2) above seems to be new.

Let $U \rightarrow V$ be a minimal resolution of singularities.

Claim 1. $\left|-K_{U}\right|$ has a reduced irreducible member. Here we do not need the assumptions that $\rho(V)=1$ and $\pi_{1}\left(V^{0}\right)=(1)$.

Recall that by Kawamata-Viehweg vanishing theorem the group $H^{1}\left(U, 2 K_{U}\right)=(0)[\mathrm{KMM}$, Theorem 1-2-3]. Hence by the Riemann-Roch theorem, one has $\operatorname{dim}\left|-K_{U}\right|=K_{U}^{2}$. Suppose that a member $A$ of $\left|-K_{U}\right|$ contains an arithmetic genus $\geq 1$ irreducible component $A_{0}$. The RiemannRoch theorem implies that $\left|A_{0}+K_{U}\right| \neq \emptyset$. From this and $0=A+K_{U}=$ $\left(A_{0}+K_{U}\right)+\left(A-A_{0}\right)$, we deduce that $A_{0}=A$ with $p_{a}\left(A_{0}\right)=1$ and Claim 1 is true.

So we may assume that every member of $\left|-K_{U}\right|$ is a union of smooth rational curves. The Stein factorization and the fact that $q(U)=0$ imply 
that a general member of $\left|-K_{U}\right|$ is of the form $M_{1}+\cdots+M_{k}+F$, where $F$ is the fixed part of the linear system, $M_{i} \cong \mathbf{P}^{1}$, and $M_{i} \sim M_{j}$.

Suppose that $K_{U}^{2}=1$. If $F=0$, then $k=1$ and $M_{1}^{2}=1$ and Claim 1 is true. Since $-K_{U}$ is nef and big, it is 1-connected by a result of C.P. Ramanujam. Hence $1=K_{U}^{2} \geq\left(k M_{1}+F\right) \cdot k M_{1} \geq 1+k^{2} M_{1}^{2}$. Thus $M_{1}^{2}=0, k=1, M_{1} \cdot F=1, K_{U} \cdot F=0$. Now intersecting the relation $-K_{U} \sim M_{1}+F$ with the smooth rational curve $M_{1}$ of self intersection 0 , one gets a contradiction. So Claim 1 is true when $K_{U}^{2}=1$.

For $K_{U}^{2} \geq 2$, let $U_{1} \rightarrow U$ be the blow-up of a point on $M_{1} \backslash\left(M_{2}+\right.$ $\left.\cdots+M_{k}+F\right)$. Then $-K_{U_{1}}$ is linearly equivalent to the proper transform of $M_{1}+\cdots+M_{k}+F$ and hence nef and big. If $U_{1} \rightarrow V_{1}$ is the contraction of all (-2)-curves then $V_{1}$ is a Gorenstein log del Pezzo surface. For $U_{1}$, we argue as in the case of $U$ and we can reduce the proof of Claim 1 to the case $K_{U}^{2}=1$, which has been dealt with in the previous paragraph. This proves Claim 1.

We continue the proof of Lemma 4. Suppose again first that $K_{U}^{2}=1$. Then $\operatorname{dim}\left|-K_{U}\right|=1$ and hence $\left|-K_{U}\right|$ has a single base point, say $p$. Let $A_{1}, A_{2}$ be two general members of $\left|-K_{U}\right|$ meeting at $p$. Let $Y \rightarrow U$ be the blow-up of $p$ with $E$ the exceptional curve. Then $Y$ has a relatively minimal elliptic fibration $Y \rightarrow \mathbf{P}^{1}$ with the proper transforms of $A_{1}, A_{2}$ as fibres and $E$ as a section.

Claim 2. (1) The singular fibre type of $Y \rightarrow \mathbf{P}^{1}$ is $I I^{*}+I I$ or $I I^{*}+$ $I_{1}+I_{1}$. Hence $Y=X$ or $Y=X^{\prime}$ as described earlier in this section.

(2) The section $E$ is the only (-1)-curve on $Y$. All (-2)-curves are in the type $I I^{*}$ fibre. There are no other negative curves on $Y$.

By the assumption of Lemma 4, Pic $V$ is of rank 1. Since $V$ is simply connected, Pic $V$ is also torsion free. Since $K_{V}^{2}=1$, one has Pic $V=\mathbf{Z} K_{V}$. The assumption that $\pi_{1}\left(V^{0}\right)=(1)$ implies that the Weil divisor class group $\operatorname{Div}(V)$ is torsion free and of rank 1 so that $\operatorname{Div}(V)=\mathbf{Z} C$ for some divisor $C$. Write $C=a K_{V}$ with a rational number $a \leq 1$. Then $a=C \cdot K_{V}$ is an integer. So $a=1$ and $\operatorname{Div}(V)=\operatorname{Pic} V$. Note that $\operatorname{Div}(U)$ is the direct sum of the pull back of $\operatorname{Div}(V)$ and the lattice generated by components of the exceptional divisor of the resolution $U \rightarrow V$. Now the unimodularity of $\operatorname{Div}(U)$ implies that $V$ has exactly one singularity and it is of type $E_{8}$.

Clearly, the fibre on $Y$ containing the inverse of the type $E_{8}$ divisor on $U$ (contractible to the singular point on $V$ ) is of type $I I^{*}$. There are no other reducible fibres by noting that $\rho(Y)=10$ and that the section $E$, a 
general fibre and the 8 components in the type $I I^{*}$ fibre which is of Dynkin type $E_{8}$, already give rise to 10 linearly independent classes of $\operatorname{Div}(Y)$. The fact that the Euler number of $Y$ is 12 implies that the singular fibre type of the elliptic fibration $Y \rightarrow \mathbf{P}^{1}$ is $I I^{*}+I I$ or $I I^{*}+I_{1}+I_{1}$. This proves part (1) of Lemma 4 and part (1) of Claim 2.

Note that this also proves that $V_{8}, V_{8}^{\prime}$ are the only rank 1 , Gorenstein log del Pezzo surfaces with $K_{V}^{2}=1$.

Let $E_{1}$ be another $(-1)$-curve on $Y$. Then the observation that $-K_{Y} \cdot E_{1}=1$ and the fact that $-K_{Y}$ is linearly equivalent to an elliptic fibre $F$ by Kodaira's canonical bundle formula imply that $E_{1}$ is a section of the elliptic fibration. Hence we can write $E_{1}=E+a F+D$ where $a$ is rational and $D$ supported by the type $E_{8}$ divisor in the type $I I^{*}$ fibre. Since $D^{2}<0$ and $D \cap E=\phi$ when $D \neq 0$, one has $D=0$ by using $D$ to intersect the expression of $E_{1}$. This leads to $-1=(E+a F)^{2}=-1+2 a$ and $a=0$, a contradiction. This proves (2) of Claim 2.

In view of what has been proved so far, we will assume that $2 \leq d \leq 6$. Denote $9-d$ by $c$. From $d \leq 6$ we get $c \geq 3$.

Claim 3. There is a composition of blow-ups $U_{7} \rightarrow U_{6} \rightarrow \cdots \rightarrow U_{c}=$ $U$, so that if $U_{i} \rightarrow W_{i}(c \leq i \leq 7)$ is the contraction of all $(-2)$-curves then $W_{i}$ is a rank 1 , Gorenstein log del Pezzo surface with $\pi_{1}\left(W_{i}^{0}\right)=(1)$ and $K_{W_{i}}^{2}=9-i$.

Let $A$ be an irreducible member of $\left|-K_{U}\right|$ and $E_{c}$ a $(-1)$-curve on $U$. Such a (-1)-curve exists because $K_{V}^{2}<7$ and hence $U$ is not a relatively minimal surface. Then $A$ meets $E_{c}$ at a point $q$. Let $U_{c+1} \rightarrow U$ be the blow-up of $q$ with $E_{c+1}$ the exceptional curve. Then $-K_{U_{c+1}}$ is linearly equivalent to the proper transform $A_{c+1}$ of $A$. Since $K_{c+1}^{2}>0$, the divisor $-K_{c+1}$ is nef and big. Moreover, the curves having 0 intersection with $A_{c+1}$ are precisely the inverse images of the $(-2)$-curves on $U$ (contractible to singular points on $V$ ) and the proper transform $E_{c}^{\prime}$ of $E_{c}$. It follows that the contraction of all the $(-2)$-curves on $U_{c+1}$ gives a surface $W_{c+1}$ as in the first part of Claim 3.

We will prove that $\pi_{1}\left(W_{c+1}^{0}\right)=(1)$. Let $\Gamma$ denote the union of the $(-2)$-curves in $U_{c}$. Then $\Gamma$ can be considered as a divisor on $U_{c+1}$ since $A$ is disjoint from $\Gamma$. By assumption, $\pi_{1}\left(U_{c}-\Gamma\right)=\pi_{1}\left(U_{c+1}-\Gamma\right)$ is trivial. Consider the natural map $\pi_{1}\left(U_{c+1}-\Gamma-E_{c}^{\prime}\right) \rightarrow \pi_{1}\left(U_{c+1}-\Gamma\right)=(1)$. By an application of Van Kampen theorem, we see that the kernel of this map is the normal subgroup of $\pi_{1}\left(U_{c+1}-\Gamma-E_{c}^{\prime}\right)$ generated by a small loop 
around $E_{c}^{\prime}$. Since $E_{c+1}$ intersects $E_{c}^{\prime}$ transversally once, this loop can be taken to be in $E_{c+1}$. But $E_{c+1}-\left(\Gamma \cup E_{c}^{\prime}\right) \cong \mathbf{C}$. Hence this loop is trivial in $\pi_{1}\left(U_{c+1}-\Gamma-E_{c}^{\prime}\right)$. This proves that $\pi_{1}\left(U_{c+1}-\Gamma-E_{c}^{\prime}\right)$ is trivial.

We will now use the assertions of Claims 2 and 3 to complete the proof of Lemma 4.

We will first prove that any rank 1, Gorenstein log del Pezzo surface $V$ with simply-connected smooth locus and $K_{V}^{2}=2$ is isomorphic to the surface $V_{7}$ constructed earlier in this section. We will show that $\left|-K_{U}\right|$ contains a cuspidal rational curve $C$ (of arithmetic genus 1). Again let $A$ be an irreducible member of $\left|-K_{U}\right|$ and $L$ a $(-1)$-curve on $U$. Let $U_{8}$ be the blow-up of $A \cap L$. The contraction of all the $(-2)$-curves in $U_{8}$ produces either $V_{8}$ or $V_{8}^{\prime}$, since these are the only surfaces with $d=1$. If $U_{8}$ is the minimal resolution of $V_{8}$ then we already know that $\left|-K_{U_{8}}\right|$ contains a cuspidal curve. In fact, we know in this case by Claim 2 that there is a unique $(-1)$-curve in $U_{8}$ and the contraction of this curve is the minimal resolution of $V_{7}$. In this case $V \cong V_{7}$. So assume that $U_{8}$ is the minimal resolution of $V_{8}^{\prime}$. Recall that $X^{\prime}$ is obtained by resolving the base locus of a pencil in $\mathbf{P}^{2}$ generated by a nodal cubic $B^{\prime}$ and 3 times a line tangent at an inflexion point of the cubic.

By the proof of Claim 2, the first 7 blow-downs of $(-1)$-curves starting from $E$ are unique, viz. the curves $E, C_{1}, \ldots, C_{6}$ in this order. The contraction of $E$ produces $U_{8}$. Hence the morphism $X^{\prime} \rightarrow \mathbf{P}^{2}$ factors as $X^{\prime} \rightarrow U \rightarrow \mathbf{P}^{2}$. The existence of a cuspidal curve $C \in\left|-K_{U}\right|$ is equivalent to the existence of a cuspidal cubic $B$ in $\mathbf{P}^{2}$ which has the same inflexion point and local intersection number 7 at the inflection point with $B^{\prime}$. We can assume that the equation of $B^{\prime}$ is $\left\{Y^{2} Z=X^{3}+X Z^{2}+\sqrt{-4 / 27} Z^{3}\right\}$, the point $[0,1,0]$ as the inflexion point, the tangent line being $\{Z=0\}$. We can then take $B$ to be the cubic $\left\{Y^{2} Z=X^{3}\right\}$. Now we see immediately that the blow-up of the point $B \cap L$ is the minimal resolution of $V_{8}$. Then again $V$ is the surface $V_{7}$.

Now by Claim 2 we easily deduce that any rank 1, Gorenstein log del Pezzo surface with simply-connected smooth locus and $3 \leq K^{2} \leq 7$ is one of the surfaces $V_{i}, 3 \leq i \leq 7$. Consider the degree 6 hypersurface $Z_{a}=\left\{W^{2}+Z^{3}+X^{5} Y+a X^{4} Z=0\right\}$ in the weighted projective space $\mathbf{P}(1,1,2,3)$ with coordinates $X, Y, Z, W$ of weights $1,1,2,3$ respectively.

Lemma 5. When $a=0$ (resp. $a \neq 0), Z_{a}$ is isomorphic to $V_{8}$ (resp. $\left.V_{8}^{\prime}\right)$.

Proof. The affine open subset $\{X \neq 0\}$ of $Z_{a}$ is isomorphic to $\mathbf{C}^{2} . Z_{a}$ 
has a unique singularity of type $E_{8}$ at $[0,1,0,0]$ (see $\left.[6]\right)$. It is easy to see that the boundary curve $\left\{X=0=W^{2}+Z^{3}\right\}$ is isomorphic to a cuspidal cubic in $\mathbf{P}^{2}$; in particular it is irreducible and hence $Z_{a}$ has rank 1 . If $a=0$ then the curve $C:=\{Y=0\}$ is a cuspidal rational curve and does not pass through the singular point of $Z_{0}$. Futher, we have $K_{Z_{0}} \sim-C$. Hence $Z_{0}$ is isomorphic to $V_{8}$. If $a \neq 0$ then $C:=\{Y=0\}$ does not pass through the singular point of $Z_{a}$. The curve $C=\left\{W^{2}+Z^{3}+a X^{4} Z=0\right\}$ in $\mathbf{P}(1,2,3)$ is easily seen to be a smooth elliptic curve and $K_{Z_{a}} \sim-C$. In [6] it is shown that the local rings of $Z_{0}$ and $Z_{a}$ at their singular points are not isomorphic. Hence $Z_{a}$ is isomorphic to $V_{8}^{\prime}$. This completes the proof of lemma 5 .

We have also proved parts (i), (ii) of Theorem 2. The part (iii) is shown in the construction of $V_{i}$, noting that $d=K_{V_{i}}^{2}=9-i$.

\section{Proof of part (iv)}

Recall that $C$ is a cuspidal rational curve on $V_{i}$ not passing through the singular point of $V_{i}$, where $3 \leq i \leq 8$ and $-K_{V_{i}} \sim C$. Then $C^{2}=9-i$. By blowing up $V_{i}$ minimally at the singular point of $C$ we get a normal crossing divisor with smooth rational irreducible components $\cup_{0}^{3} B_{i}$ on a normal projective surface $V_{i}^{\prime \prime}$, where $B_{0}^{2}=-1, B_{1}^{2}=-2, B_{2}^{2}=-3, B_{3}^{2}=3-i, B_{0}$ intersects $B_{1}, B_{2}, B_{3}$. The curves $B_{1}, B_{2}, B_{3}$ are mutually disjoint and $B_{3}$ is the proper transform of $C$. Mumford's presentation for the fundamental group $G$ of the boundary of a nice tubular neighborhood of $\cup B_{i}$ is as follows (see $[10])$.

$$
\left\langle e_{2}, e_{3} \mid\left(e_{2} e_{3}\right)^{2}=e_{2}^{3}=e_{3}^{i-3}\right\rangle
$$

Now assume that $4 \leq i \leq 8$. Then this group is finite. This is the fundamental group at infinity of the affine surface $V_{i}-C$.

Lemma 6. For $4 \leq i \leq 8$ the surface $V_{i}-C$ is isomorphic to $\mathbf{C}^{2} / G$.

Proof. Denote the surface $V_{i}-C$ by $S$. We have proved above that $S$ has a unique singular point, say $p$. First we treat the case $d=1$. As seen above, in this case $S$ is isomorphic to the affine subset $\left\{W^{2}+Z^{3}+X^{5}=0\right\}$ of the projective surface $\left\{W^{2}+Z^{3}+X^{5} Y=0\right\}$ considered above given by $\{Y \neq 0\}$. It is a classical fact that this $S$ is isomorphic to the quotient of $\mathbf{C}^{2}$ modulo the binary icosahedral group of order 120. Since $d=1$, the fundamental group at infinity of $S$ has the presentation $<e_{2}, e_{3} \mid\left(e_{2} e_{3}\right)^{2}=e_{2}^{2}=e_{3}^{5}>$. This group is the binary icosahedral group. 
Now we assume that $4 \leq i \leq 7$.

Claim. For a small nice neighborhood $U$ of $p$ in $V_{i}$, the natural homomorphism $\pi_{1}(U-\{p\}) \rightarrow \pi_{1}(S-\{p\})$ is an isomorphism.

Proof. We will illustrate the proof by giving the argument for $d=2$. Other cases are dealt in exactly the same way. The surface $V_{7}$ is obtained from the elliptic surface $X$ by contracting the curves $E, C_{1}$ and all the other irreducible components of the type $I I^{*}$ fiber $F_{0}$ except for $C_{2}$, giving rise to an $E_{7}$-singularity. Let $D$ denote the inverse image of $p$ in $X$. Clearly, $S-\{p\}=X-\left(C \cup E \cup C_{1} \cup D\right)$. Let $N$ denote a suitable tubular neighborhood of $F_{0}$ in $X$. It is easy to see that $N-\left(E \cup C_{1} \cup D\right)$ is a strong deformation retract of $X-\left(C \cup E \cup C_{1} \cup D\right)$. The neighborhood $N$ is a union of tubular neighborhoods $N_{1}, N_{2}, N_{D}$ of $C_{1}, C_{2}, D$ respectively. Since $E \cap C_{1}$ is a single point $\left(N_{2} \cup N_{D}\right)-\left(C_{1} \cup D\right)$ is a strong deformation retract of $N-\left(E \cup C_{1} \cup D\right)$. Since $C_{1} \cap C_{2}$ is a single point $N_{D}-D$ is a strong deformation retract of $\left(N_{2} \cup N_{D}\right)-\left(C_{1} \cup D\right)$. But $N_{D}-D$ is nothing but $U-\{p\}$. This proves the claim.

Let $W^{\prime}$ denote the universal covering space of $S-\{p\}$ and let $W$ be the normalization of $S$ in the function field of $W^{\prime}$. By the claim just proved, $W$ contains a unique point, say $q$, over $p$. This point is smooth by the claim just proved. Since $V_{7}$ has rank 1 we see that $\chi(S-\{p\})=0$ where $\chi$ denotes the topological Euler number. Therefore, $\chi\left(W^{\prime}\right)=0$ and hence $\chi(W)=1$. Now $W$ is smooth, simply-connected and $b_{2}(W)=0$, hence it is contractible.

Using $-K_{V_{7}} \sim C$ we see easily that the canonical bundle of $V_{7}^{\prime \prime}$ is linearly equivalent to a strictly negative linear combination of the curves $B_{0}, B_{1}, B_{2}, B_{3}$. It follows that $\bar{\kappa}(S-\{p\})=-\infty$. The map $W^{\prime} \rightarrow S-$ $\{p\}$ being unramified and proper we get $\bar{\kappa}\left(W^{\prime}\right)=-\infty$. This implies that $\bar{\kappa}(W)=-\infty$. Since $W$ is contractible, by a fundamental result of MiyanishiSugie-Fujita, $W$ is isomorphic to $\mathbf{C}^{2}$. This easily implies that $S \cong \mathbf{C}^{2} / G$, as desired. This completes the proof of Theorem 2 .

\section{$\S 4$. Proof of Theorem 1}

Let $V$ be a Gorenstein normal projective surface such that there is a surjective morphism $f: \mathbf{P}^{2} \rightarrow V$. We claim that $\pi_{1}\left(V^{\circ}\right)$ is finite, where $V^{\circ}=V \backslash \operatorname{Sing} V$. Since $f^{-1}(\operatorname{Sing} V)$ has codimension 2 in $\mathbf{P}^{2}$, the complement $\mathbf{P}^{2} \backslash f^{-1}(\operatorname{Sing} V)$ is simply-connected. If $W^{\prime}$ is the universal cover 
of $V^{\circ}$, then by the standard properties of topological coverings the restriction of $f$ factors through $\mathbf{P}^{2} \backslash f^{-1}(\operatorname{Sing} V) \rightarrow W^{\prime}$. This easily proves the claim. Let $W \rightarrow V$ be the quasi-universal cover of $V$. Then $f$ factors as $\mathbf{P}^{2} \rightarrow W \rightarrow V$. Now $\pi_{1}\left(W^{\circ}\right)=(1)$ and $W$ is Gorenstein of rank 1. From now onwards we will assume that $V^{\circ}$ is simply-connected.

First we deal with the case $d=1$.

Assume that $V=V_{8}$. We will show that there is no non-constant morphism $\mathbf{P}^{2} \rightarrow V_{8}$.

Consider the surface $V_{4}$. Let $p$ be the singular point and $C$ the cuspidal rational curve on $V_{4}^{0}$ such that $K_{V_{4}} \sim-C$. As in Theorem 2, one has $C \sim 5 \Delta$, where $\Delta$ is the image of the curve $C_{5}$ in $X$. By Theorem 2 we have a quotient map $\alpha$ of degree $5, \mathbf{C}^{2} \rightarrow V_{4}-C$. Since $\alpha$ is proper, a neighborhood of infinity of $\mathbf{C}^{2}$ maps onto a neighborhood of infinity of $V_{4}-C$. Since $\mathbf{C}^{2}$ is simply connected at infinity, we can use an argument similar to the one at the beginning of this section to show that the order of the fundamental group at infinity of $V_{4}-C$ is at most 5 . There is a unique point, say $\tilde{p}$, in $\mathbf{C}^{2}$ over $p$. Let $Z$ be the normalization of $V_{4}$ in the function field of $\mathbf{C}^{2}$. We will show that $Z$ is isomorphic to $V_{8}$.

Since $V_{4}^{0}$ is simply-connected, the curve $C$ is ramified. Hence its inverse image in $Z$, say $D$, is irreducible and maps homeomorphically onto $C$. If $q$ is the singular point of $C$ then the local analytic equation of $C$ at $q$ is $z_{1}^{2}+z_{2}^{3}=0$. Hence the equation of $Z$ at its point $\tilde{q}$ over $q$ is $z_{1}^{2}+z_{2}^{3}+z_{3}^{5}=0$. This is the only singular point of $Z$ and it is clearly an $E_{8}$-singularity. As $Z$ contains $\mathbf{C}^{2}$, we see that $Z$ is a rank 1 , Gorenstein $\log$ del Pezzo surface such that $\pi_{1}\left(Z^{0}\right)=(1)$. To see that $Z$ is isomorphic to $V_{8}$ we argue as follows.

There is a unique point in $Z$ over $C \cap \Delta$. Since $\Delta-\{p\}-(C \cap \Delta) \cong \mathbf{C}^{*}$, we can prove easily that the inverse image of $\Delta$ in $Z$, say $\tilde{\Delta}$, is irreducible and rational and it is smooth outside $\tilde{p}$. Note that on $V_{4}$ we have $-K_{V_{4}} \sim$ $5 \Delta \sim C$. From the simple-connectedness of $Z^{0}$ we deduce that on $Z$ we have $D \sim \tilde{\Delta}$. This implies that on $Z$ there is a cuspidal rational curve $\tilde{\Delta}$ contained in the smooth locus of $Z$ such that $-K_{Z} \sim \tilde{\Delta}$. By Theorem 2 we infer that $Z$ is isomorphic to $V_{8}$. As remarked above we will show that there is no non-constant morphism $\mathbf{P}^{2} \rightarrow V_{4}$. This will prove that $V_{8}$ is not dominated by $\mathbf{P}^{2}$.

The cases $d=2,3,4,5$. 
First consider the cases $d=3,4,5$. By Lemma 2, $\left|-K_{V}\right|$ embeds $V$ in $\mathbf{P}^{d}$ and the image is projectively normal. Let $T \subset \mathbf{C}^{d+1}$ be the cone over this embedding. The map $T \backslash\{$ vertex $\} \stackrel{\sigma}{\rightarrow} V$ is a locally trivial $\mathbf{C}^{*}$ bundle. It is the associated principal bundle of the line bundle $\mp K_{V}$ over $V$. The sign does not play any significant role in our argument so we use the negative sign. This gives the exact sequence

$$
\pi_{1}\left(\mathbf{C}^{*}\right) \rightarrow \pi_{1}\left(T^{\circ}\right) \rightarrow \pi_{1}\left(V^{\circ}\right) \rightarrow(1)
$$

where $T^{\circ}=T \backslash \operatorname{Sing} T$. Since $\pi_{1}\left(V^{\circ}\right)=1$, we see that $\pi_{1}\left(\mathbf{C}^{*}\right) \rightarrow \pi_{1}\left(T^{\circ}\right)$ is surjective.

LEMma 7. The fundamental group of $T^{\circ}$ is isomorphic to $\mathbf{Z} /(d)$.

Proof. Let $C \subset V^{\circ}$ be the cuspidal rational curve with $C \sim-K_{V}$. Then $C^{2}=d$. The inverse image $\sigma^{-1}(C)$ is the total space of the principal bundle over $C$ of the line bundle $-\left.K_{V}\right|_{C}$ which has degree $d$. If $\bar{C} \stackrel{\beta}{\rightarrow} C$ is the normalization then $\beta$ is a homeomorphism. The pull-back of the $\mathbf{C}^{*}$-bundle on $C$ to $\bar{C}$ is a $\mathbf{C}^{*}$-bundle of degree $d$. Since $\overline{\mathbf{C}} \cong \mathbf{P}^{1}$, this pullback has fundamental group isomorphic to $\mathbf{Z}_{d}$. It follows that $\pi_{1}\left(T^{\circ}\right)$ is a homomorphic image of $\mathbf{Z}_{d}$.

We will now construct a cyclic $d$-fold étale cover of $T^{\circ}$. Since $\pi_{1}\left(V^{\circ}\right)=$ (1) and rank of $V$ is one, the class group of $V$ is cyclic. Let $\Delta$ be the generator of the Weil divisor class group as given in Theorem 2, so that $-K_{V} \sim d \Delta$ in $\operatorname{Div}(V)$, or $\mathcal{O}\left(-K_{V}\right) \cong \mathcal{O}(\Delta)^{\otimes d}$ restricted to $V^{\circ}$. We can find a suitable covering $\left\{U_{i}\right\}$ of $V^{\circ}$ of Euclidean balls and transition functions $f_{i j}$ for $\left.\mathcal{O}(\Delta)\right|_{V^{\circ}}$ such that $f_{i j}^{d}$ are the transition functions for $\left.\mathcal{O}\left(-K_{V}\right)\right|_{V^{\circ}}$. If $\mathcal{T} \rightarrow V^{\circ}$ is the total space of the associated $\mathbf{C}^{*}$-bundle for $\mathcal{O}(\Delta)$, then the maps $U_{i} \times \mathbf{C}^{*} \rightarrow U_{i} \times \mathbf{C}^{*}$ defined by $(z, \lambda) \mapsto\left(z, \lambda^{d}\right)$ patch to give an étale cover of $T \backslash \operatorname{Sing} T$ of degree $d$. This proves the lemma.

The map $\mathbf{P}^{2} \rightarrow V \subset \mathbf{P}^{d}$ gives rise to a finite morphism $\mathbf{C}^{3} \stackrel{\pi}{\rightarrow} T$. If $W \rightarrow T$ is the quasi-universal $\mathbf{Z} /(d)$-cover then $\pi$ factors as $\mathbf{C}^{3} \rightarrow W \stackrel{\tau}{\rightarrow} T$. By lemma 3, $W$ admits an action of $\mathbf{C}^{*}$. This is easily seen to be a good $\mathbf{C}^{*}$-action such that $\mathbf{C}^{3} \rightarrow W$ is a $\mathbf{C}^{*}$-equivariant map. For a general fiber $F$ of $\sigma$ in $T^{\circ}, \tau^{-1}(F)$ is smooth and irreducible because $\pi_{1}\left(\mathbf{C}^{*}\right) \rightarrow \pi_{1}\left(T^{\circ}\right)$ is surjective. Further, $(W \backslash\{$ vertex $\}) / / \mathbf{C}^{*} \cong V$.

We claim that the coordinate ring $\Gamma(W)$ of $W$ is a UFD. Since the map $\mathbf{C}^{3} \rightarrow W$ is proper we see that $\operatorname{Div}(W)$ is finite. Any non-trivial torsion 
line bundle on $W^{0}$ gives rise to a non-trivial topological covering of $W^{0}$. But $\pi_{1}(W \backslash \operatorname{Sing} W)=(1)$. Hence $\Gamma(W)$ is a UFD. Recall that $d=3,4$ or 5. Then $G / \Gamma \cong \mathbf{Z} /(d)$, where $\Gamma=[G, G]$ and $G$ is defined in Lemma 6 .

Let $Z$ be the inverse image of $V \backslash C$ in $W \backslash\{$ vertex . Then $Z \backslash l$ is the total space of the principal $\mathbf{C}^{*}$-bundle associated to the line bundle $\left.\mathcal{O}(\Delta)\right|_{V \backslash C}$, where $l$ is the inverse image in $Z$ of the singular point of $V$. Consider the following action of $G$ on $\mathbf{C}^{2} \times \mathbf{C}^{*}$. We consider the surjection $\mu: G \rightarrow G / \Gamma(\cong \mathbf{Z} /(d))$. Let $\bar{g}$ be a generator of $G / \Gamma$ and $g$ a lift of $\bar{g}$ in $G$. Any element of $G$ has the unique expression $\gamma \cdot g^{b}, \gamma \in \Gamma$ and $0 \leq b \leq d-1$. Let $h=\gamma g^{b}$ act on $(z, \lambda)$ by $h(z, \lambda)=\left(h z, \omega^{b} \lambda\right)$, where $\omega=\exp (2 \pi i / d)$.

LEMMA 8. With the above action we have $\left(\mathbf{C}^{2} \times \mathbf{C}^{*}\right) / G \cong Z$.

Proof. Recall that the inverse image of $V \backslash C$ in $T$ is isomorphic to $(V \backslash C) \times \mathbf{C}^{*} \approx\left(\mathbf{C}^{2} / G\right) \times \mathbf{C}^{*}$. This is because $\left.K_{V}\right|_{V \backslash C}$ is a trivial line bundle. Define the map $\alpha: \mathbf{C}^{2} \times \mathbf{C}^{*} \rightarrow\left(\mathbf{C}^{2} / G\right) \times \mathbf{C}^{*}$ given by $(z, \lambda) \mapsto\left(\bar{z}, \lambda^{d}\right)$. If $\gamma g^{b}$ is an arbitrary element of $G$ as above, then $\alpha\left(\left(\gamma g^{b}\right)(z, \lambda)\right)=\left(\bar{z}, \lambda^{d}\right)$. Hence the map $\alpha$ factors through $\bar{\alpha}:\left(\mathbf{C}^{2} \times \mathbf{C}^{*}\right) / G \rightarrow\left(\mathbf{C}^{2} / G\right) \times \mathbf{C}^{*}$. If $q \in \mathbf{C}^{2} / G$ is a smooth point of $\mathbf{C}^{2} / G$ then $\alpha^{-1}(q, \lambda)$ has $d|G|$ points. Hence $\bar{\alpha}^{-1}(q, \lambda)$ has $d$ points and $\bar{\alpha}$ is étale outside $\{\overline{0}\} \times \mathbf{C}^{*}$. We see easily that the inverse image of an orbit $q \times \mathbf{C}^{*}$ in $\left(\mathbf{C}^{2} \times \mathbf{C}^{*}\right) / G$ is connected. On the other hand, for the map $W \backslash\{$ vertex $\} \rightarrow T \backslash\{$ vertex $\}$, we have proved that any good orbit $q \times \mathbf{C}^{*} \subset T \backslash\{$ vertex $\}$ lifts to a single orbit in $W \backslash\{$ vertex $\}$. From these two observations, we deduce that $\mathbf{C}^{2} \times \mathbf{C}^{*} / G$ is naturally isomorphic to $Z$, proving the lemma.

We now come to the punch line (deducing a contradiction to the fact that the affine open subset $Z \subseteq W$ also has a UFD as the coordinate ring).

LEMma 9. The affine 3-fold $\left(\mathbf{C}^{2} \times \mathbf{C}^{*}\right) / G$ is not a $U F D$.

Proof. The map $\mathbf{C}^{2} \times \mathbf{C}^{*} \rightarrow\left(\mathbf{C}^{2} \times \mathbf{C}^{*}\right) / G$ is unramified outside $\{\overline{0}\} \times$ $\mathbf{C}^{*}$, which has codimension 2 . Let $U$ be the group of units in the coordinate $\operatorname{ring} R$ of $\mathbf{C}^{2} \times \mathbf{C}^{*}$. Then $U \cong \mathbf{C}^{*} \times \mathbf{Z}$. Here $\mathbf{C}^{*}$ is the multiplicative unit group of the underlying base field and $\mathbf{Z} t$ generates the group of units of $\mathbf{C}^{*}$ (modulo the non-zero constants). By Samuel's descent theory (see [12], Chapter III), the divisor class group of $\left(\mathbf{C}^{2} \times \mathbf{C}^{*}\right) / G$ is $H^{1}(G, U)$. Consider the short exact sequence of $G$-modules

$$
(1) \rightarrow \mathbf{C}^{*} \rightarrow U \rightarrow \mathbf{Z} t \rightarrow(1)
$$


Here, we consider $U / \mathbf{C}^{*} \cong \mathbf{Z} t$ as a $G$-module. The long exact cohomology sequence corresponding to this looks like

$$
\begin{aligned}
(1) \rightarrow H^{0}\left(G, \mathbf{C}^{*}\right) \rightarrow H^{0}(G, U) & \rightarrow H^{0}(G, \mathbf{Z} t) \\
& \rightarrow H^{1}\left(G, \mathbf{C}^{*}\right) \rightarrow H^{1}(G, U) \rightarrow \cdots
\end{aligned}
$$

Now $H^{0}(G, \mathbf{Z} t)$ is the cyclic subgroup of $\mathbf{Z} t$ of index $d$ invariant under $G$ and $H^{0}(G, U)$ is a direct sum $H^{0}\left(G, \mathbf{C}^{*}\right) \oplus H^{0}(G, \mathbf{Z} t)$. Hence $H^{1}\left(G, \mathbf{C}^{*}\right)$ is a subgroup of $H^{1}(G, U)$. On the other hand, since $G$ acts trivially on the field of constants $\mathbf{C}, H^{1}\left(G, \mathbf{C}^{*}\right)=\operatorname{Hom}\left(G, \mathbf{C}^{*}\right) \cong \operatorname{Hom}\left(G / \Gamma, \mathbf{C}^{*}\right) \cong \mathbf{Z} /(d)$. But $d \neq 1$ by assumption. This proves the lemma.

So we have proved that $V$ cannot be an image of $\mathbf{P}^{2}$, for those $V=V_{i}$ with $d=K_{V_{i}}^{2}=9-i=1,3,4,5$.

Next we consider the cases $d=2,6,8$.

The case $d=2$.

In this case $\left|-K_{V}\right|$ does not give an embedding of $V$ but $\left|-2 K_{V}\right|$ gives a projectively normal embedding of $V$ in $\mathbf{P}^{6}$. Let $T$ denote the affine cone over this embedding. Then we work with this cone $T$. An easy modification of the argument for $3 \leq d \leq 5$ shows that $V_{i}(i=9-d=7)$ is not an image of $\mathbf{P}^{2}$.

The case $d=6$.

In this case $V \backslash C$ is not isomorphic to $\mathbf{C}^{2} / G$. In fact $V \backslash C$ has singularity type $A_{1}+A_{2}$. Consider the action of $\mathbf{Z}_{6}$ on $\mathbf{P}^{2}$ given by $\sigma\left[x_{0}, x_{1}, x_{2}\right]=$ $\left[x_{0}, \omega x_{1},-x_{2}\right]$ where $\omega=\exp (2 \pi i / 3)$. Let $x, y$ be suitable affine coordinates on $\mathbf{P}^{2} \backslash\left\{x_{0}=0\right\}$. Then $\sigma(x, y)=(-x, \omega y)$ and $\sigma^{2}(x, y)=\left(x, \omega^{2} y\right)$ which implies that $\sigma^{2}$ is a pseudo-reflection. Similarly $\sigma^{3}$ is a pseudo-reflection. Hence $\mathbf{C}^{2} /\langle\sigma\rangle$ is smooth and hence isomorphic to $\mathbf{C}^{2}$.

Consider $\mathbf{P}^{2} \backslash\left\{x_{1}=0\right\}$. Now $\sigma(x, y)=\left(\omega^{2} x,-\omega^{2} y\right)$ and $\sigma^{3}(x, y)=$ $(x,-y)$ and hence $\sigma^{3}$ is a pseudo-reflection. The ring of invariants of $\sigma^{3}$ is $\mathbf{C}\left[x, y^{2}\right]$. The action of $\bar{\sigma}$ on $\mathbf{C}\left[x, y^{2}\right]$ is $\left(x, y^{2}\right) \mapsto\left(\omega^{2} x, \omega y^{2}\right)$. This gives an $A_{2}$-singularity.

Finally consider $\mathbf{P}^{2} \backslash\left\{x_{2}=0\right\}$. In this case $\sigma(x, y)=(-x,-\omega y)$ which implies that $\sigma^{2}(x, y)=\left(x, \omega^{2} y\right)$. The invariants are $\mathbf{C}\left[x, y^{3}\right]$ and hence $\bar{\sigma}\left(x, y^{3}\right)=\left(-x,-y^{3}\right)$. This gives an $A_{1}$-singularity on the quotient.

By Theorem $2, \mathbf{P}^{2} /\langle\sigma\rangle=V_{3}$ where $d=K_{V_{3}}^{2}=9-3=6$. In other words, $V_{3}(d=6)$ is the quotient of $\mathbf{P}^{2}$ by an action of $\mathbf{Z} /(6)$.

The case $d=8$. 
In this case $V$ is the quadric cone $Q$ in $\mathbf{P}^{3}$. Let $\mathbf{Z} /(2)$ act on $\mathbf{P}^{2}$ by $\sigma\left[x_{0}, x_{1}, x_{2}\right]=\left[-x_{0},-x_{1}, x_{3}\right]$. Then $V \cong \mathbf{P}^{2} / \mathbf{Z} /(2)$.

In conclusion, we have so far proved that if $V$ is a rank 1 , Gorenstein $\log$ del Pezzo surface such that $\pi_{1}\left(V^{0}\right)$ is trivial and there is a non-constant morphism $\mathbf{P}^{2} \rightarrow V$ then $V$ is isomorphic to either $\mathbf{P}^{2}, Q$, a surface of singularity type $A_{1}+A_{2}$, or $V_{8}^{\prime}$. The values of $d$ in these cases are 9,8,6,1 respectively. We will next prove that if $V$ is a rank 1 , Gorenstein log del Pezzo surface dominated by $\mathbf{P}^{2}$ such that $\pi_{1}\left(V^{0}\right)$ is non-trivial then its quasi-universal cover is either $\mathbf{P}^{2}$ or $Q$. Let $W$ be the quasi-universal cover of $V$ and $g: W \rightarrow V$ the covering map. Then $W$ is a rank 1 Gorenstein, $\log$ del Pezzo surface dominated by $\mathbf{P}^{2}$ such that $\pi_{1}\left(W^{\circ}\right)=(1)$.

Assume first that $K_{W}^{2}=1$. Since $K_{W} \sim g^{*} K_{V}$, we get $K_{W}^{2}=1=\operatorname{deg} g \cdot K_{V}^{2}$. This means that $g$ is an isomorphism. Hence $V_{8}, V_{8}^{\prime}$ cannot occur as the quasi-universal cover of $V$.

Suppose that $W$ is of singularity type $A_{1}+A_{2}$. Let the singular points of $W$ be $p, q$. As $g$ is unramified over $V^{\circ}$, the images $p^{\prime}:=g(p), q^{\prime}:=g(q)$ are singular points of $V$. Also, $6=K_{W}^{2}=(\operatorname{deg} g) K_{V}^{2}$. We analyse the possible cases.

Case 1. Suppose that $\operatorname{deg} g=2$. Then any singular point of $V$ other than $p^{\prime}, q^{\prime}$ is an $A_{1}$-singular point. Let $\tilde{V} \rightarrow V$ be a minimal resolution of singularities. Since $K_{V}^{2}=3$ and $\rho(V)=1$, the number of irreducible exceptional curves for the map $\tilde{V} \rightarrow V$ is 6 . It is easy to see that $p^{\prime}, q^{\prime}$ are of type $A_{3}, A_{5}$ respectively. This is a contradiction.

Case 2. Suppose that $\operatorname{deg} g=3$. Then $K_{V}^{2}=2$ and the number of exceptional curves for $\tilde{V} \rightarrow V$ is 7 . The type of $p^{\prime}$ is $A_{5}$ and $q^{\prime}$ will give rise to more than 2 exceptional curves. This is a contradiction.

Case 3. Suppose that $\operatorname{deg} g=6$. Now $K_{V}^{2}=1$ and the number of exceptional curves is 8 . The local fundamental groups at $p^{\prime}, q^{\prime}$ have orders 12,18 respectively. Looking at the possible Dynkin types of these singularities we arrive at a contradiction.

This proves part (2) of Theorem 1.

\section{Proof of Part (3).}

As above, let $f: \mathbf{P}^{2} \rightarrow V$ be a non-constant morphism. We will assume that $V$ is not $V_{8}^{\prime}$. Assume that the quasi-universal cover of $V$ is $Q$. We will prove that $V$ is isomorphic to $\mathbf{P}^{2} / H$ for a suitable finite group 
of automorphisms $H$ of $\mathbf{P}^{2}$. Of course this $\mathbf{P}^{2}$ may not be the same as the projective plane dominating $V$.

Let $q$ be the singular point of $Q$. It is easy to see that $Q$ contains a smooth rational curve $D$ with $D^{2}=2$ and not passing through $q$. Further, $\pi_{1}(Q-D-\{q\})=\mathbf{Z} /(2)$. If $Y$ is the universal cover of $Q-D-\{q\}$ then the normalization of $Q$ in the function field of $Y$ is isomorphic to $\mathbf{P}^{2}$ such that the inverse image of $D$ in $\mathbf{P}^{2}$ is a line. We will use this observation below.

By assumption, $V$ is isomorphic to $Q / G$ with $G=\pi_{1}\left(V^{0}\right)$, and the map $Q \rightarrow V$ is unramified over $V^{0}$. Then $K_{Q}^{2}=8=|G| K_{V}^{2}$. Therefore $|G|$ is of order 2,4 or 8 . The action of $G$ extends uniquely to the minimal resolution of singularities of $Q$, viz. to Hirzebruch surface $\Sigma_{2}$. Let $M$ be the unique $(-2)$-curve on $\Sigma_{2}$ and let $L$ be a fiber of the $\mathbf{P}^{1}$-fibration on $\Sigma_{2}$. Then $G$ acts naturally on $|D|$ and $D \sim M+2 L$. The linear system $|D|$ is parametrized by $\mathbf{P}^{3}$. The subspace of members of this of the form $M+2 L^{\prime}$, where $L^{\prime}$ is a fiber of the $\mathbf{P}^{1}$-fibration is parametrized by $\mathbf{P}^{2}$. This 2-dimensional subspace is clearly stable under the action of $G$. The complement of this 2-dimensional subspace in $|D|$ is parametrized by $\mathbf{C}^{3}$. As $G$ is a finite 2-group, by a standard result in Smith Theory the action of $G$ on $\mathbf{C}^{3}$ has a fixed point (see, [2]). This means that there is an irreducible smooth rational curve $D_{0} \in|D|$ which is stable under $G$. Hence the set $Q-D_{0}-\{q\}$ is also $G$-stable. This implies that the map $Q \rightarrow V$ is unramified over $Q-D_{0}-\{q\}-A_{2}$ with a finite set $A_{2}$ and $Q-D_{0}-\{q\}-A_{2}$ is the full inverse image of its image in $V$. From the observation made in the beginning, we conclude that there is a line $C$ in $\mathbf{P}^{2}$ such that the composite $\operatorname{map} \mathbf{P}^{2}-C-\left\{q_{1}\right\}-A_{1} \rightarrow V-E-\left\{q_{3}\right\}-A_{3}$ is unramified, where $E, q_{3}, A_{3}$ (resp. $\left.C, q_{1}, A_{1}\right)$ are images (resp. inverse images) of $D, q, A_{2}$ in $V$ (resp. $\left.\mathbf{P}^{2}\right)$. Since $\mathbf{P}^{2}-C-\left\{q_{1}\right\}-A_{1}$ is simply-connected, the morphism $\mathbf{P}^{2} \rightarrow V$ is a Galois map.

To complete the proof of part (3), we will now show that $V_{8}^{\prime}$ is not isomorphic to a quotient $\mathbf{P}^{2} / G$. Suppose that $V=V_{8}^{\prime} \cong \mathbf{P}^{2} / G$ and let $f: \mathbf{P}^{2} \rightarrow V$ be the quotient map and $\operatorname{deg} f=n$. Let $\Gamma_{1}, \ldots, \Gamma_{m}$ be the irreducible components of the branch locus in $V$. Denote by $\Gamma_{i j}$ the irreducible components of $f^{-1} \Gamma_{i}$ with ramification index $e_{i}$. Then $f^{*} \Gamma_{i}=$ $\Sigma_{j} e_{i} \Gamma_{i j}$. For the canonical bundle we have $K_{\mathbf{P}^{2}} \sim f^{*} K_{V}+\Sigma_{i, j}\left(e_{i}-1\right) \Gamma_{i j}$. Write $\Gamma_{i} \sim \delta_{i} C$, where $C$ is an irreducible curve on $V$ such that $K_{V} \sim-C$. This gives $K_{\mathbf{P}^{2}} \sim \Sigma_{i} f^{*}\left(\frac{e_{i}-1}{e_{i}} \delta_{i}-1\right) C$. Since $K_{\mathbf{P}^{2}}$ is negative, we infer easily that the branch curve in $V$ is irreducible and $\delta_{i}=1$. This means that the 
branch curve is a member of $\left|-K_{V}\right|$. But it can be easily seen from the arguments used earlier that for any member $D$ of $\left|-K_{V}\right|$ the complement $V \backslash D$ is simply-connected. This shows that $V_{8}^{\prime}$ is not a quotient of $\mathbf{P}^{2}$. This also completes the proof of Theorem 1 .

\section{§5. Classification of Gorenstein quotients of $\mathbf{P}^{2}$}

Let $V=\mathbf{P}^{2} / G$ be a normal Gorenstein quotient of $\mathbf{P}^{2}$.

Case 1. The quotient map $f: \mathbf{P}^{2} \rightarrow V$ is a quasi-universal covering.

In this case $9=(\operatorname{deg} f) K_{V}^{2}$. Hence $|G|=3$ or 9.

Case 1.1. Suppose that $|G|=3$.

In this case every singular point of $V$ is an $A_{2}$-singularity. Let $\tilde{V} \rightarrow V$ be the minimal resolution of singularities. Since $K_{V}^{2}=3$, the number of irreducible components of the exceptional divisor for the map $\tilde{V} \rightarrow V$ is 6 . This means that $V$ has exactly three singular points. It is easy to see that in a suitable coordinate system, the action of a generator of $G$ is given by $[X, Y, Z] \rightarrow\left[X, \omega Y, \omega^{2} Z\right]$, where $\omega$ is a primitive cubic root of unity.

Case 1.2. Suppose that $|G|=9$.

Now $K_{V}^{2}=1$. The number of irreducible components of the exceptional divisor for the map $\tilde{V} \rightarrow V$ is 8 . The map $f$ is unramified outside finitely many points. Hence the order of the local fundamental group at any singular point of $V$ divides 9 . First, we claim that $A_{8}$ cannot occur as a singularity of $V$. For otherwise $V$ has no other singularity and the map $f$ is unramified outside the singular point and there is a unique singular point in $\mathbf{P}^{2}$ over this point. But $\chi\left(\mathbf{P}^{2} \backslash\{\right.$ one point $\left.\}\right)=2=\chi(V \backslash\{$ one point $\})$. This contradicts the multiplicativity of $\chi$ for topological coverings. We deduce that $V$ has exactly four $A_{2}$-type singular points. There are exactly three distinct points in $\mathbf{P}^{2}$ over each of these. We claim that $G$ is isomorpic to a direct $\operatorname{sum} \mathbf{Z} /(3) \oplus \mathbf{Z} /(3)$ so that $\pi_{1}\left(V^{0}\right) \cong \mathbf{Z} /(3) \oplus \mathbf{Z} /(3)$. Assume that $G$ is cyclic, say $G=\langle g\rangle$. We can assume that the action of $g$ sends $[X, Y, Z] \rightarrow\left[\omega X, \omega^{q} Y, Z\right]$ for some integer $q$, where $\omega=\exp (2 \pi \imath / 9)$. The point $[0,0,1]$ is fixed under the group and the action of $G$ near this point cannot have any non-trivial pseudo-reflection as the map $f$ is divisorially unramified. Since the singularities in $V$ are Gorenstein we deduce that $q=8$. But this produces an $A_{8}$-type singularity. This proves the claim.

Now we will construct an explicit example giving such a surface. Let $G=\mathbf{Z} /(3) \oplus \mathbf{Z} /(3)=\left(g_{1}\right) \oplus\left(g_{2}\right)$, where $g_{1}$ sends $[X, Y, Z] \mapsto\left[X, \omega Y, \omega^{2} Z\right]$ where $\omega$ is a primitive cube root of unity and $g_{2}$ sends $[X, Y, Z] \mapsto[Z, X, Y]$. 
Let $W=\mathbf{P}^{2} /\left(g_{1}\right)$. The points $[1,0,0],[0,1,0]$ and $[0,0,1]$ are fixed by $g_{1}$ and form a single $g_{2}$-orbit. Their images in $W$ are $A_{2}$ type singular points. The points $[1,1,1],\left[1, \omega, \omega^{2}\right]$ and $\left[1, \omega^{2}, \omega\right]$ are fixed by $g_{2}$ and form a single $g_{1}$-orbit. Their image in $W$ is another $A_{2}$ type singular point. The points $[1,1, \omega],[1, \omega, 1]$ and $\left[1, \omega^{2}, \omega^{2}\right]$ form both a single $g_{1}$-orbit and a single $g_{2}$-orbit. Their image in $V$ is a singular point of type $A_{2}$. Finally $\left[1, \omega^{2}, 1\right]$, $\left[1,1, \omega^{2}\right],[1, \omega, \omega]$ form both a single $g_{1}$-orbit and a single $g_{2}$-orbit. Their image in $V$ is the fourth singular point of type $A_{2}$ in $V$.

Case 2. $h: Q \rightarrow V$ is the quasi-universal covering. Let $H$ be the Galois group.

Let $q$ be the singular point of $Q$. In this case, $8=(\operatorname{deg} g) K_{V}^{2}$.

Case 2.1. Suppose that $|H|=2$.

Since $K_{V}^{2}=4$ the number of exceptional irreducible components for $\tilde{V} \rightarrow V$ is 5 . The image of $q$ is of $A_{3}$-type. All other singular points are of $A_{1}$-type. Thus $V$ has singularity type $A_{3}, A_{1}, A_{1}$. By Theorem 1 , $V \cong \mathbf{P}^{2} / G$ with $|G|=4$. The existence of type $A_{3}$ singularity on $V$ shows that $G \cong \mathbf{Z} /(4)$.

An explicit example in this case is given as follows. Let $g$ be the automorphism of $\mathbf{P}^{2}$ of order 4 sending $[X, Y, Z] \mapsto[X, \imath Y,-\imath Z]$ where $\imath$ is a square root of -1 . The point $[1,0,0]$ is fixed by $g$ and every point on $X=0$ is fixed by $g^{2}$. No other point of $\mathbf{P}^{2}$ has a non-trivial isotropy group. The image of $[1,0,0]$ on $V$ is an $A_{3}$ type singularity and the images of $[0,1,0]$ and $[0,0,1]$ on $V$ are $A_{1}$ type singular points. The quotient $\mathbf{P}^{2} /\left(g^{2}\right)$ is the quadric $Q$.

Case 2.2. Suppose that $|H|=4$.

Now $K_{V}^{2}=2$. The number of irreducible exceptional curves for $\tilde{V} \rightarrow V$ is 7. The image of $q$ in $V$, say $q^{\prime}$, has local fundamental group of order 8. If $q^{\prime}$ is of type $A_{7}$ then $V$ has no other singular points. But this contradicts the multiplicativity of $\chi$.

Hence $q^{\prime}$ is of $D_{4}$-type. Then the other singular points of $V$ are of type $A_{1}, A_{2}$ or $A_{1}, A_{1}, A_{1}$. But the order of the local fundamental group of any other singular point is a divisor of 4 . Hence we conclude that $V$ has singularity type $D_{4}+3 A_{1}$. By Theorem $1, V \cong \mathbf{P}^{2} / G$ with $|G|=8$. The existence of the type $D_{4}$ singularity on $V$ shows that $G$ is the binary dihedral group of order 8. An explict action of $G$ is as follows. Let $g_{1}$ map $[X, Y, Z] \mapsto[X, \imath Y,-\imath Z]$ and $g_{2}$ map $[X, Y, Z] \mapsto[X, \imath Z, \imath Y]$. Then $g_{1}, g_{2}$ generate a group of order 8 such that the subgroup $\left(g_{1}\right)$ has index 2 and 
hence normal in $G$. Arguing as in case 1.2 by considering the intermediate quotient $W=\mathbf{P}^{2} /\left(g_{1}\right)$ we see that $V$ has singularity type $D_{4}+3 A_{1}$.

Case 2.3. Suppose that $|G|=8$.

Now $K_{V}^{2}=1$ and the number of irreducible exceptional curves for $\tilde{V} \rightarrow V$ is 8 . The image $q^{\prime}$ of $q$ has local fundamental group of order 16 . This cannot be of $A_{15}$-type. Hence $q^{\prime}$ is of type $D_{6}$. The other singular points are of type $A_{2}$ or $A_{1}, A_{1}$. Again $A_{2}$ cannot occur because the order of the local fundamental group is not a divisor of 8 . Hence $V$ is of singularity type $D_{6}+2 A_{1}$. We claim that this case cannot occur. Over a singular point of type $A_{1}$ there are four points in $Q$. This easily contradicts the multiplicativity of $\chi$.

Finally, we consider the surface $V_{3}$ of singularity type $A_{1}+A_{2}$. An explicit action of $\mathbf{Z} /(6)$ on $\mathbf{P}^{2}$ which produces this quotient is as follows. Let $g$ be an automorphism of $\mathbf{P}^{2}$ of order 6 sending $[X, Y, Z] \mapsto[X,-Y, \omega Z]$, where $\omega$ is a primitive cube root of unity. The image of $[0,1,0]$ in $V$ is an $A_{1}$ type singularity. The image of $[0,0,1]$ in $V$ is an $A_{2}$ type singularity.

We have thus proved the following result.

LEMMA 10. If $\mathbf{P}^{2}$ is the quasi-universal cover of a normal, Gorenstein, projective surface $V$ (not isomorphic to $\mathbf{P}^{2}$ ) then either $V^{0}$ has fundamental group $\mathbf{Z} /(3)$ and singularity type $3 A_{2}$, or the fundamental group of $V^{0}$ is $\mathbf{Z} /(3) \oplus \mathbf{Z} /(3)$ and $V$ is of singularity type $4 A_{2}$.

If $Q$ is the quasi-universal cover of a normal projective Gorenstein surface $V$ (not isomorphic to $Q$ ) then either the order of the fundamental group of $V^{0}$ is $2, V$ is of singularity type $A_{3}+2 A_{1}$ and $V \cong \mathbf{P}^{2} / \mathbf{Z} /(4)$, or the order of the fundamental group of $V^{0}$ is $4, V$ is of singularity type $D_{4}+3 A_{1}$ and $V \cong \mathbf{P}^{2} / H$ with $H$ the quaternion group of order 8 .

The only other Gorenstein surface not covered by the above cases which is isomorphic to a quotient of $\mathbf{P}^{2}$ is the surface $V_{3}$ of singularity type $A_{1}+$ $A_{2}$. The fundamental group of $V_{3}^{0}$ is trivial and $V_{3} \cong \mathbf{P}^{2} / \mathbf{Z} /(6)$.

\section{§6. Log del Pezzo non-Gorenstein case}

Assume that $f: \mathbf{P}^{2} \rightarrow V$ is surjective and $V$ is a $\log$ del Pezzo surface such that $\pi_{1}\left(V^{\circ}\right)=(1)$. Let $V \subset \mathbf{P}^{N}$ be a suitable projectively normal embedding and $T$ the cone over $V$ in $\mathbf{C}^{N+1}$. Then we get a finite map 
$\mathbf{C}^{3} \rightarrow T$. Denote by $W$ the quasi-universal cover of $W$. As before $T$ admits a good $\mathbf{C}^{*}$-action such that the map $\mathbf{C}^{3} \rightarrow T$ is $\mathbf{C}^{*}$-equivariant.

ConjeCture 1. Let $Y$ be a normal affine variety with a good $\mathbf{C}^{*}$ action. Suppose $\pi: \mathbf{C}^{n} \rightarrow Y$ is a proper surjective morphism which is $\mathbf{C}^{*}$-equivariant. Assume that $\pi_{1}(Y \backslash \operatorname{Sing} Y)=(1)$. Then $Y$ is isomorphic to $\mathbf{C}^{n}$ with a suitable good $\mathbf{C}^{*}$-action.

If this conjecture has an affirmative answer then $Y / / \mathbf{C}^{*}$ is a weighted projective space $\mathbf{P}(a, b, c, \ldots)$. In the case of log del Pezzo surface under consideration, we know that $W / / \mathbf{C}^{*} \cong V$. Hence $V$ is isomorphic to $\mathbf{P}(a, b, c)$. Therefore an affirmative answer to the above conjecture gives an affirmative answer to

Conjecture 2. Let $V$ be a log del Pezzo surface with a surjective morphism $f: \mathbf{P}^{2} \rightarrow V$. Then $V$ is isomorphic to a quotient $\mathbf{P}(a, b, c) / G$, with $G$ isomorphic to the fundamental group $\pi_{1}\left(V^{\circ}\right)$.

In particular, if $V^{\circ}$ is simply-connected then $V$ is isomorphic to $\mathbf{P}(a, b, c)$.

Remark. In the Gorenstein case, $d=8$ corresponds to $\mathbf{P}(1,1,2)$ and $d=6$ to $\mathbf{P}(1,2,3)$.

\section{REFERENCES}

[1] Alekseev, V. A. and Kolpakov-Miroshnichenko, I. Ya., On quotient surfaces of $\mathbf{P}^{2}$ by a finite group, Russian Math. Surveys, 43-5 (1988), 207-208.

[2] Bredon, G. E., Introduction to Compact Transformation Groups, Pure and Applied Mathematics, Vol. 46, Academic Press, 1972.

[3] Demazure, M., Surfaces de del Pezzo, Lecture Notes in Math. Vol. 777, Springer, Berlin-Heidelberg-New York, 1980.

[4] Hidaka, F. and Watanabe, K., Normal Gorenstein surfaces with ample anti-canonical divisor, Tokyo J. Math., 4 (1981), 319-330.

[5] Gurjar, R. V. and Shastri, A. R., The fundamental group at infinity of affine surfaces, Comment. Math. Helvitici, 59 (1984), 459-484.

[6] Mohan Kumar, N., Rational double points on a rational surface, Invent. Math., 65 (1981/82), 251-268.

[7] Lazarsfeld, R., Some applications of the theory of positive vector bundles, Complete intersections, Acireale, Lecture Notes in Mathematics, Vol. 1092 (1983), 29-61, Springer Verlag.

[8] Miyanishi, M., Normal Affine Subalgebras of a Polynomial Ring, Algebraic and Topological Theories - to the memory of T. Miyata, 37-51, Kinokuniya, Tokyo, (1985). 
[9] Miyanishi, M. and Zhang, D. -Q., Gorenstein log del Pezzo surfaces of rank one, J. Algebra, 118 (1988), 63-84.

[10] Mumford, D., The topology of normal singularities of an algebraic surface and a criterion for simplicity, Publ. Math. IHES, 9 (1961), 5-22.

[11] Seshadri, C. S., Quotient spaces modulo reductive algebraic groups, Ann. Math., 97 (1972), 511-556.

[12] Samuel, P., On Unique Factorization Domains, Tata Institute of Fundamental Research, Lectures on Mathematics and Physics, No. 30.

R. V. Gurjar

School of Mathematics

Tata Institute of Fundamental research

Homi-Bhabha road

Mumbai 400005

India

gurjar@math.tifr.res.in

C. R. Pradeep

Department of Mathematics

Indian Institute of Science

Bangalore, 560012

India

pradeep@math.iisc.ernet.in

D.-Q. Zhang

Department of Mathematics

National University of Singapore

2 Science Drive 2

Singapore 117543

matzdq@math. nus.edu.sg 